\title{
M-mode echocardiographic study in dogs
}

\author{
M. B. Gugjoo*, A. C. Saxena, M. Hoque and M. M. S. Zama \\ Division of Surgery, Indian Veterinary Research Institute, Izatnagar, India.
}

Accepted 18 December, 2013

\begin{abstract}
Echocardiography is a unique non-invasive application of ultrasound for imaging of living heart. It is based on detection of echoes produced by a beam of ultrasound pulses transmitted into the heart. Conventional echocardiographic modalities include two-dimensional (2D), M-mode and Doppler modes. M-mode is the first ultrasound modality used in which the ultrasound beams are aimed manually at selected cardiac structures to give a graphic recording of their positions and movements. M-mode recordings permit quantitative measurement of cardiac dimensions and detailed analysis of complex motion patterns depending on transducer angulation. It facilitates analysis of time relationships with other physiological variables such as echocardiographic, heart sounds, and pulse tracings, which can be recorded simultaneously. However, it cannot be used to measure velocity, the direction or type of the blood flow but can be combined with contrast or colour-coded Doppler studies for accurate timing of flow events. Variations in M-mode echocardiographic parameters with breed, age, sex and body weight occurs and need to be kept in mind while interpreting the findings. Its variables are usually subjected to change and needs experienced sonologist to diagnose any condition. The present review covers the M-mode echocardiographic developmental history in general and its diagnostic role for dogs in particular.
\end{abstract}

Key words: M-mode, echocardiography, dogs, ultrasound.

\section{INTRODUCTION}

Echocardiography, cardiac ultrasound, is an important diagnostic tool in cardiology which has been introduced in the veterinary medicine as a non-invasive method for evaluating the anatomy and function of heart (Boon, 1998). An ultrasound examination of heart and large vessels represent a significant technological advance in veterinary medicine. Echocardiography allows an evaluation of the space relationship between structures, cardiac movement and blood flow features, the precise and non-invasive diagnosis of cardiac alterations, as well as follow-up therapy and to determine the prognosis through direct vision of cardiac chambers (Gugjoo et al., 2013a). It allows assessment of cardiac chamber sizes, cardiac function and blood flow all of which provide information on hemodynamic status and extent of disease process together with follow up therapy (Boon,
1998). Defects which can be visualized including valvular lesions (Bonagura and Schober, 2009), cardiac shunts (Kittleson, 1998), cardiac and thoracic masses, pleural and pericardial effusions (Gugjoo et al., 2013b), myocardial diseases (Gugjoo et al., 2013a), stenotic lesions, congenital and vegetative anomalies (Boon, 1998; Bonagura, 1983). Therefore, it is important that an echocardiographic examination be considered as part of a thorough cardiovascular examination viz. clinical radiographic and electrocardiographic examination.

Ability of ultrasonic waves to distinguish between fluid and soft tissues (unlike conventional radiography); to define spatial relationships between structures and to detect quantitative motion has made it a particularly valuable tool in cardiovascular diagnosis (Feigenbaum, 1981). As ultrasound images can discriminate between 
blood filled cardiac chambers and soft tissue structures of heart while radiographs can distinguish lungs from soft tissues and fluid density, echo images are complimentary to radiographic images for cardiac assessment. When complimented with electrocardiographic data, one can arrive at a conclusive diagnosis (Gugjoo et al., 2011).

Echocardiographic examination includes both qualitative as well as quantitative cardiac assessment. For quantitative examination, M-mode echocardiography is primarily being utilised for dimensional measurements and subsequently the functional activities of heart are being calculated. Reliable, normal echocardiographic values for chamber size, wall dimensions and myocardial function are needed for comparison and evaluation of dogs suspected for having heart diseases (O'Grady et al., 1986).

Earlier studies established normal reference values of echocardiographic parameters in general dog and cat population (Lombard, 1984; Jacobs and Knight, 1985). Later on studies reported the influence of body weight, body surface area and heart rate on selected echocardiographic measurements in both dogs and cats as previously described for humans. As echocardiographic values show significant breed variations, it is important to know the normal echocardiographic value for each breed of dog (Thomas et al., 1993; O'Leary et al., 2003). Therefore, currently stress is being given for breed specific studies as echocardiographic reference ranges derived from some breed of dog may be misleading to other dog breed (Snyder et al., 1995; Jacobson et al., 2013).

\section{Historical background}

Ultrasound history can be correlated with the advent of piezoelectric crystals (basic units for ultrasound production) discovered by Curie and Curie. It was observed that if piezoelectric crystals are compressed, an electric charge is produced between opposite surface and if electric potential is applied to such crystals, compression and decompressed rare fraction occurs depending upon the polarity of electric charge and thus very high frequency sound is produced (Curie and Curie, 1880). After a gap of around 70 years, Kiedel in 1950 first used transmitted ultrasound waves through the heart and recorded the acoustic shadow on the other side of the chest. He found that changes in cardiac volume would cause change in the acoustic shadow. In 1952, the first two-dimensional ultrasound unit appeared (Weyman, 1982) and in 1954, Edler and Hertz initiated the use of pulsed, reflected ultrasound for the examination of heart. They were able to identify the signal that moved with cardiac action and started in earnest, the era of time motion mode. They found this signal to be coming from anterior leaflet of mitral valve on their retrospective study on autopsy investigation, contradicting their initial thought of signal coming from posterior left atrial wall. Later, Wild et al. (1957) used the reflected ultrasound to examine the autopsy specimen of heart.

In 1973, instrumentation for satisfactory clinical use of ultrasound in cardiac investigation was developed. In 1977, M-mode as a clinically useful tool in veterinary medicine was described (Boon, 1998). Since then it has developed into a prominent diagnostic tool in veterinary cardiology. Earlier approach to M-mode echocardiography was invasive and employed implanted catheters and catheter tipped transducers, removal or displacement of lung lobes and transducer placement directly on cardiac surface (Bishop et al., 1969; Franklin et al., 1977). Transesophageal and transcutaneous were two popular invasive echocardiographic techniques with transesophageal echogram first reported by Frazin et al. (1976). In transesophageal technique, distance from the canine tooth to the fourth intercostal space was measured and the oesophageal probe transducer was introduced through oesophagus to this site, which was considered a good indicator of mitral valve location (Dennis et al., 1978).

M-mode echocardiography provide a non- invasive method of evaluating cardiac chamber size, interventricular septum, left ventricular free wall thickness, systolic and diastolic function (Calvert and Brown, 1986). Jacobs and Mahjoob (1988a) recorded Mmode echocardiograms from 10 conscious, clinically normal dogs at various heart rates during atrial pacing. Heart rate was recorded as cycle length (seconds), and measurements were made only during sustained 1:1 atrial-to-ventricular conduction. In all dogs studied, there was a significant $(P<0.01)$ positive correlation of left ventricular internal chamber dimension in diastole and systole to cycle length. Also, there was positive correlation between these left ventricular dimensions and the square root of cycle length.

The same authors adopted multiple regression analysis in dogs using body size in cardiac cycle length in predicting echocardiographic variables and found positive correlation between left ventricular internal chamber dimension in diastole and systole and body weight, body surface area, cycle length, square root of cycle length, and shortening fraction had a significant negative correlation and left ventricular free wall measurements had a significant positive correlation to body weight and body surface area. For these echocardiographic variables, correlation to square root of cycle length was insignificant and a multiple regression model was not helpful in developing confidence intervals. Septal wall measurements were not correlated with body weight, body surface area, cycle length, or square root of cycle length. They further found that fractional shortening and ejection fraction estimated by $\mathrm{M}$-mode measurements decreased with increased body weight (Jacobs and Mahjoob, 1988b). Lombard (1984) and Cornell et al. (2004) later reported that the various variables that could 
influence echocardiographic evaluation of systolic function include age, sex, breed, weight and co-morbid factors (hypothyroidism and hydration factors).

\section{ECHOCARDIOGRAPHIC STUDY IN CANINES}

The earliest work carried on dogs was conducted by Mashiro et al. (1976) who performed uni-dimensional Mmode echocardiography as a non- invasive tool for the quantitative study of heart. The first structure to be identified during the development of echocardiography was the mitral valve and detailed structures of mitral valves together with ventricular walls were studied (Yamamura et al., 1977). Later studies focused on these structures in normal (Dennis et al., 1978) as well as abnormal dogs (Pipers et al., 1981) affected with left sided heart failure. The normal dogs have a characteristic mitral valve motion in systole and diastole and they open in response to early rapid diastolic filling of the ventricle shortly after the completion of T-wave of electrocardiogram. The anterior leaflet inscribes an ' $M$ ' like motion during diastole with initial opening usually greater and longer than atrial systole. The posterior leaflet forms images of opposite configuration of ' $W$ ', but of lesser magnitude. There is a definite alteration of these relationships during cardiac diseases (Pipers et al., 1981). Different components of valve excursion observed were: C point - valve cooptation during ventricular systole, D point - initial opening during diatole, E point maximal opening during rapid ventricular filling, $F$ point end of rapid ventricular filling and A point - maximal opening during atrial systole (Figure 1).

The basic principles of M-mode echocardiography, including technical considerations were put forward by Bonagura (1983). He observed that normal structures of heart can be scanned by changing the angle or location of transducer. If the transducer is maintained in a constant position during the cardiac cycle, the phasic motion of cardiac structures can be recorded. The resultant record is termed as motion or M-mode echocardiogram. A year later, Thomas (1984) performed systemic studies to determine optimum transducer location and orientations for standardizing imaging of various cardiac structures to validate cardiac anatomy and function in dogs by two-dimensional and M-mode echocardiography. The two most useful transducer locations observed were the right intercostal and left intercostal locations in a laterally recumbent dog imaged from a dependent side using a slit on the table. Potential echo window for right intercostals space was found to be between 3rd and 7th intercostal space with best results in between 3rd and 5th intercostal space, 1 to $8 \mathrm{~cm}$, lateral to sternum. Left atrium was imaged best with long axis plane. Papillary muscles were seen at 4 and 6 o'clock position in the short axis view. Potential echo window for left intercostal space location was found to be between 3rd to 7th intercostal spaces with best results between 3rd to 6th intercostal spaces, 1 to $5 \mathrm{~cm}$, lateral to sternum. This location was further sub-divided between left cranial intercostal and left caudal intercostal locations. It was reported that left-sided cardiac structures were consistently easier to image as compared to right-sided cardiac structures. The structure images were validated using micro bubble laden saline injections and by insertion of intramedulary pins in to the heart of dog along the plane of ultrasound beams with subsequent necropsy confirmation of structures penetrated by pins.

Initially, reference values for the general dog population were developed but later on the effect of sex and body weight was observed. Boon et al. (1983) determined the reference ranges of echocardiographic structures and their relationship with body weight and body surface and they found that statistically significant correlation exists between body surface area and aortic, left atrial, left ventricular, septal and posterior wall dimensions and mitral valve amplitude of motion while velocity of circumferential fibre shortening, ejection time, percent systolic thickening of septum and posterior wall, percent change in minor diameter and mitral valve velocities were not statistically correlated. Later Lombard (1984) recorded M-echocardiogram from healthy dogs, awake and unsedated, in left lateral recumbent position. Echocardiographic measurements were taken and correlated with body weight using linear regression equation. The left ventricular internal dimension in systole and diastole, the left ventricular wall thickness, the aortic root dimension, and the left atrial dimension had high correlation $\left(r^{2}\right)$ ranging from 0.756 to 0.619 . The fractional shortening of the left ventricle in systole $(39 \pm 6 \%)$ and the left atrial to aortic root ratio $(0.99 \pm 0.10 \%)$ were not linearly related to body weights and had constant values.

Later O'Grady et al. (1986) studied tomographic planes for echocardiography of normal canine heart and were further elucidated and compared with that of previous studies by subsequent researchers. Normal chamber and wall dimensions were established to derive indices for normal ventricular functions and comparison of results obtained when structures were imaged and measured from different tomographic planes. The imaging planes were described using three variables relative to the transducer and interrogating beam viz. transducer location on chest wall, approximate direction of centre of beam and directions of extremities of ultrasound plane. The intracardiac structures were identified on the bases of position within chest, general anatomic appearance, motion, association with known structures and selective injection of echo dense micro bubbles. The measurements were made from the trailing endocardial edge of anterior wall to the leading endocardial edge of posterior wall for every structure.

However, later studies recommended leading edge method (Wyatt et al., 1980) in which measurements are made from leading endocardial edge of anterior wall to leading endocardial edge of posterior wall (Figure 2). 


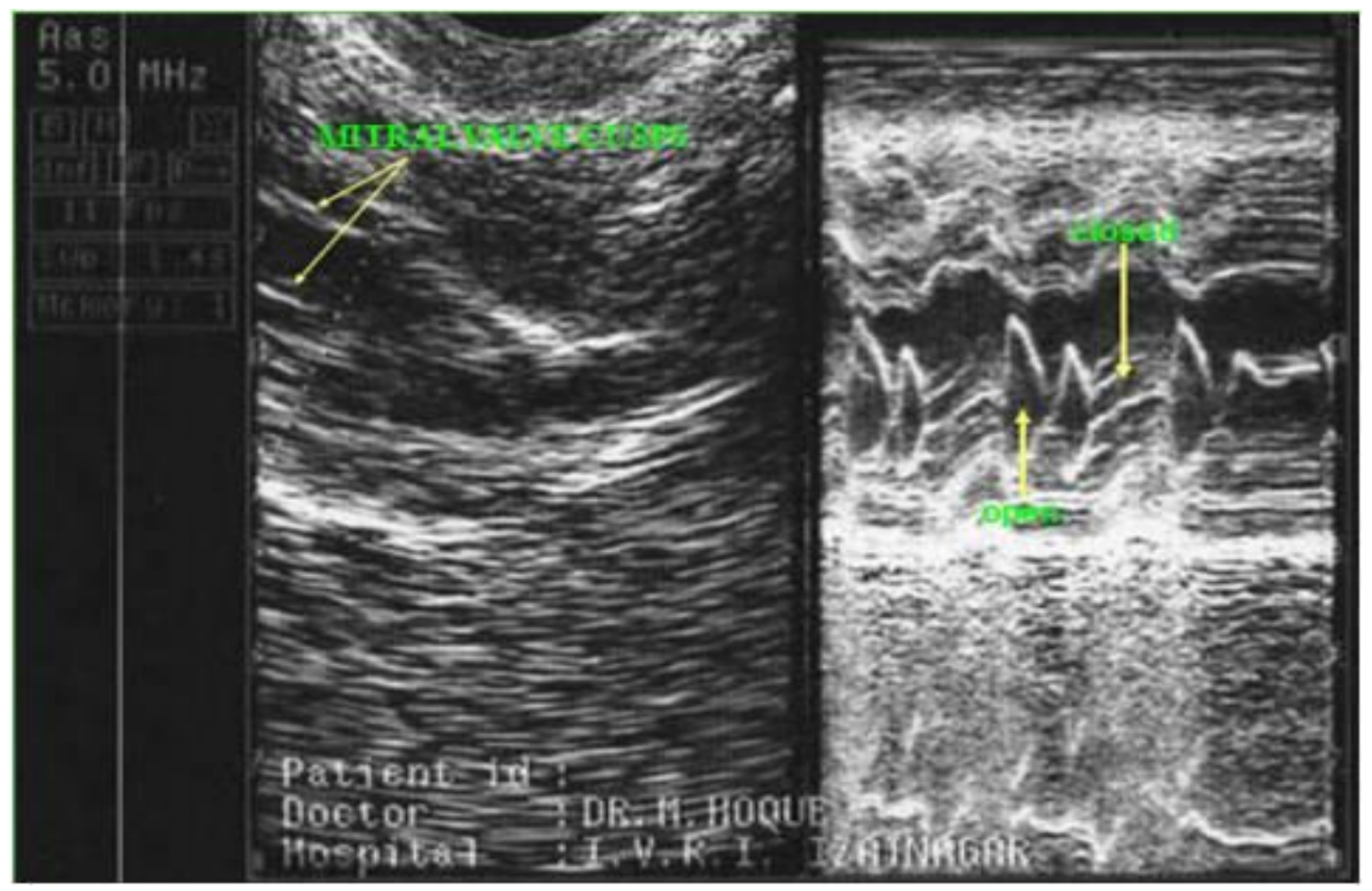

Figure 1. Mitral valve in $M$-mode showing $M$ and $W$ images of anterior and posterior valves.

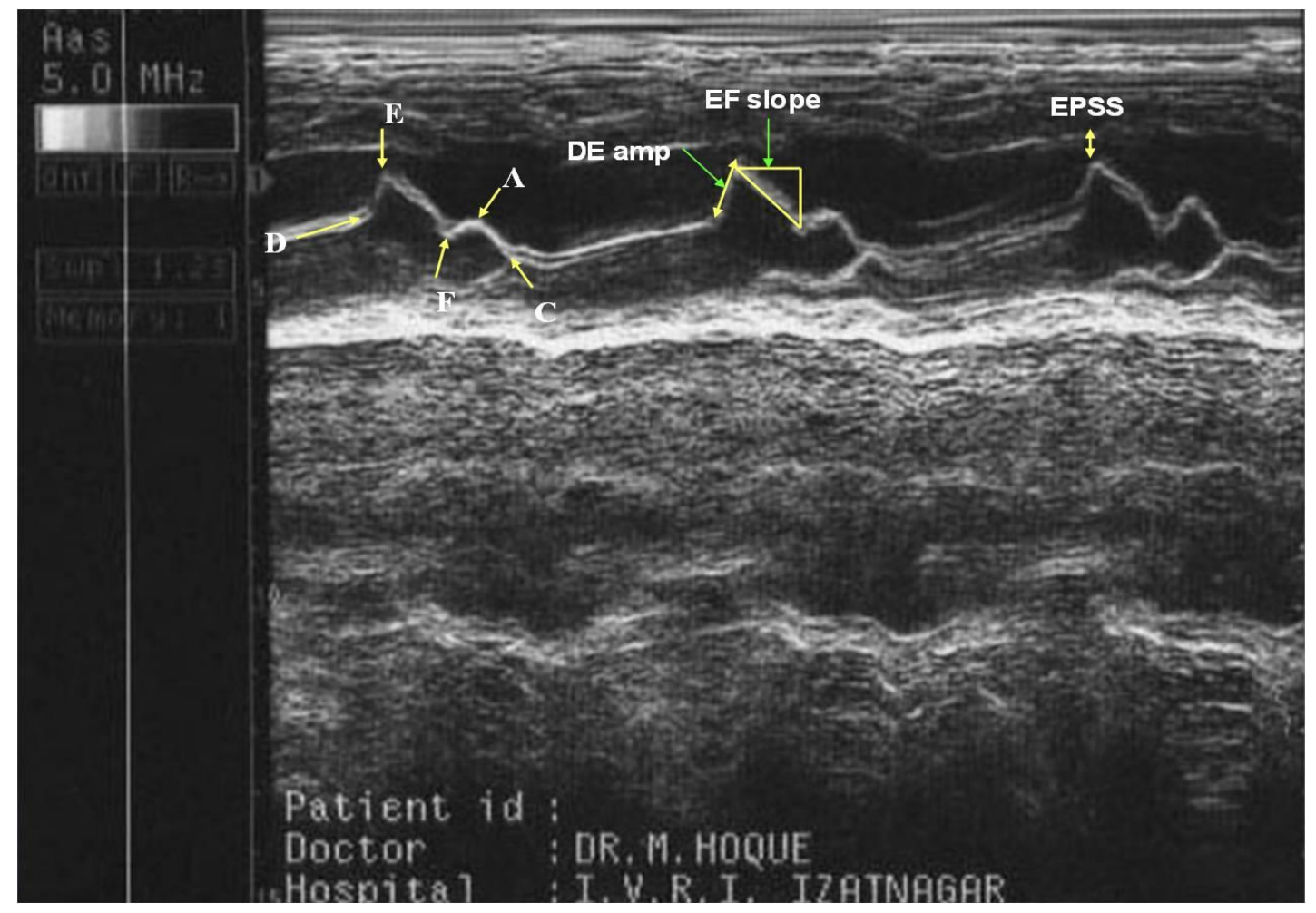

Figure 2. Marking of Mitral valve leaflets on M-mode echocardiogram: D point - initial opening during diastole; $E$ point - maximal opening during rapid ventricular filling; $F$ point - end of rapid ventricular filling; $A$ point - maximal opening during atrial systole; C point - valve cooptation during ventricular systole. 
Quantitatively different parameters that were determined include fractional shortening, left ventricular volume, stroke volume, ejection fraction, fractional thickening of left ventricular free wall \& interventricular septum and ratio of mitral valvular orifice area to left ventricular internal cavity area. It was found that all linear and area measurements were significantly correlated to body size while most indices of left ventricular function were independent of body weight.

Relative development of heart with body weight observed by M-mode echocardiography was measured by Sisson and Schaeffer (1991). Echocardiographic measurements were obtained from 16 English Pointers at $1,2,4$ and 8 weeks of age and at 3, 6, 9 and 12 months of age. Left atrial (LA), aortic (Ao), left and right ventricular internal dimensions, interventricular septal and left ventricular wall thickness increased in curvilinear fashion relative to increasing body weight. Least-squares regression analysis, performed on logarithmically transformed data, was used to develop power-law equations describing the relationship of echocardiographic measurements to body weight.

Linear dimensions of the LA, Ao, left and right ventricular internal dimensions and interventricular septal and left ventricular wall thickness changed proportionally to slightly differing exponential powers of body weight (BW), varying from 0.31 to $0.45\left(\mathrm{BW}^{0.31}\right.$ to $\left.\mathrm{BW}^{0.45}\right)$. Fractional shortening and the LA/Ao ratio decreased slightly but significantly as the body weight increased. Indexing echocardiographic measurement to $\mathrm{BW}^{1 / 3}$ was more appropriate than indexing such measures linearly to body weight, offering a practical method for developing accurate normative graphs or tables for M-mode echocardiographic dimensions on growing dogs.

With age cardiac dimensions increase with significant effect observed only on the left ventricular wall thickness after animal attains maturity (Sisson and Schaeffer, 1991; Bayon et al., 1994). Heart rate has an inverse relation with the left ventricular systolic and diastolic dimensions and left atrial size (Jacobs and Mahjoob, 1988a)

Varability of left ventricular (LV) M-mode echocardiogram in relation to axis (Long v's Short axis) in healthy and diseased dogs was observed by Schober and Baade (2000). Mean left ventricular diameter (LVD) in systole and diastole and mean interventricular septum (IVS) in systole were significantly $(P<0.001)$ larger when measured from short-axis compared to long-axis measurements. An increased magnitude of measurement resulted in increased differences between the methods for LV dimensions and fractional shortening. Differences between the two methods were small and within clinically acceptable limits in normal dogs. However, in some of the dogs with cardiac abnormality, one or more LV Mmode derived dimension obtained from one imaging plane did not agreed sufficient enough with the same measure from the other plane.

Only for measurement of FS was there good agreement between methods in dogs with cardiac disease. Therefore, with the exception of FS, data gained from LV short-axis and long-axis M-mode recordings should not be used interchangeably in dogs with cardiac disease. In the same year, four 2-dimensional echocardiographic methods were used for evaluating left atrium (LA) size in dogs viz. LA diameter in short axis, LA diameter in long axis, LA circumference in short axis, and LA cross-sectional area in short axis (Rishniw and Erb, 2000). Comparisons of these LA dimensions to appropriate aortic dimensions provided body weightindependent estimates of LA size. They observed strong associations of LA dimensions with body weight $\left(r^{2}=\right.$ 0.76-0.88). Comparable body weight-independent $2 \mathrm{D}$ echocardiographic estimates of LA size in short axis exceeded historical M-mode reference intervals.

The left ventricular volumes (end diastolic volume and end systolic volume) are being calculated by Teicholz formulae (Teicholz et al., 1976) as under:

End diastolic volume (EDV in $\mathrm{ml})=7(\mathrm{X}) \mathrm{LVDd}^{3} /(2.4+$ LVDd)

End systolic volume (ESV in $\mathrm{ml})=7(\mathrm{X}) \mathrm{LVDs}^{3} /(2.4$ +LVDs).

The rest of the parameters are calculated using established formulae (Kienle, 1998; Riedesel and Knight, 2005) as follows:

Stroke volume $($ SV in $\mathrm{ml})=\mathrm{EDV}-\mathrm{ESV}$

Ejection fraction $(\%)=($ EDV- ESV) $(X) 100 /$ EDV

Left ventricle fractional shortening $($ LVFS in $\%)=($ LVDd LVDs) (X) 100/ LVDd

To determine the reference values of M-mode echocardiogram in dogs, different models (linear, logarithmic and polynomial) were compared (Goncalves et al., 2002). Logarithmic or second- order polynomial models predicted reference values of M-mode measurements for size of the cardiac chambers were found better than simple linear models for dogs with a wide range of body weights. However, no significant differences were observed with respect to cardiac wall thickness.

Brown et al. (2003) introduced a novel method for quantitative echocardiographic interpretations based on the calculation of ratio indices in which each raw M-mode measurement was divided by the aortic root dimension (Ao) (Figure 3). "Aorta-based" indices were calculated with the animal's measured aortic root dimension (Ao (m)) as the length standard. Conversely, "weight-based" indices employed an idealized estimate of aortic dimension $(A o(w))$ with a weighted least squares linear regression against the cube root of body weight $(\mathrm{Ao}(\mathrm{w})=$ 


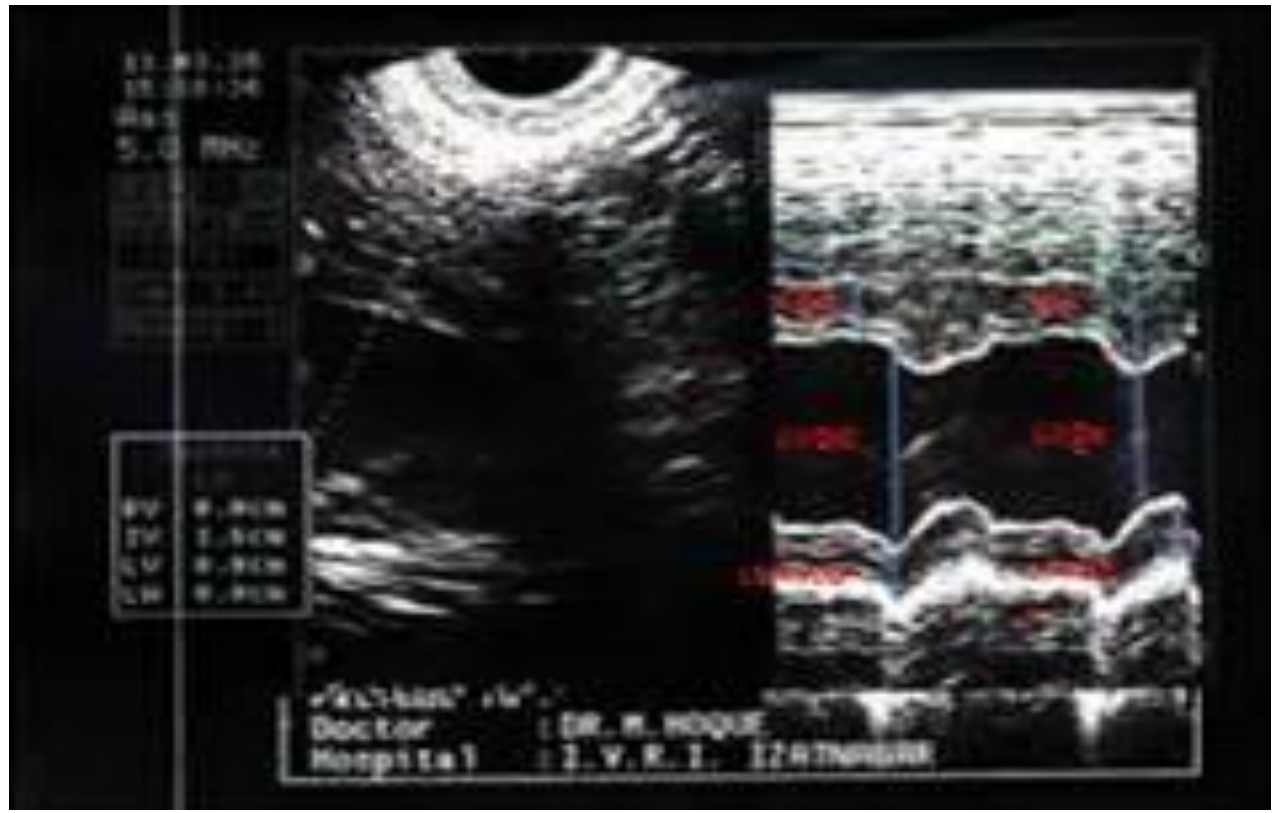

Figure 3. Measurements of left ventricular structures during systole and diastole (LVDd,s-left ventricular diameter during diastole and systole; IVSd,s - interventricular diameter during diastole and systole; LVPWd,s - left ventricular posterior wall during diastole and systole).

$\mathrm{kW}(1 / 3))$. Use of these indices circumvented undesirable statistical characteristics inherent in linear regression of echocardiographic dimensions against body weight and, to a lesser extent, body surface area. Compared with the regressions, ratio indices resulted in substantial refinement of the predictive range for each M-mode measurement in dogs, particularly with decreasing body size.

Weight-based indices outperformed aorta-based indices in this regard. To refine the predictive range, neither type of index was clearly advantageous in cats compared with the simple average method typically employed for that species. Several of the raw M-mode measurements, however, were correlated with body weight in cats and horses, indicating the need for an appropriate correction for body size in these species. The ratio index method was suitable for this purpose. Summary statistics derived from normal dogs $(n=53)$, cats $(n=32)$, and horses $(n=17)$ were presented for each index, including novel clinical indices calculated from area ratios. The latter were designed to represent body size-adjusted left ventricular stroke area (that is, volume overload) and myocardial wall area (that is, hypertrophy). This was followed by introduction of the more advanced echocardiographic technique which explained more so than other diagnostic techniques, echocardiography is highly operator dependent and relies on the proper acquisition and interpretation of results by an examiner who is familiar with the principles, capabilities, and limitations of ultrasound imaging (Oyama, 2004). He reviewed the basics of echocardiography, measurements of cardiac dimensions, and assessment of cardiac function and introduced emerging technologies that expanded the capabilities of the echocardiography examination.

Oyama and Sisson (2005) assessed cardiac chamber size using Anatomical M-mode (AMM) and compared the results of the AMM and conventional M-mode (CMM) with 2-dimensional (2D) study via linear regression and calculation of a coefficient of correlation. In healthy dogs, cardiac AMM measurements were associated with greater accuracy and less variability than CMM. AMM has potential to improve quantification of cardiac dimensions. Chetboul et al. (2005) studied the effects of animal position and number of repeated measurements on selected two-dimensional and M-mode echocardiographic variables in healthy dogs and concluded that within day variability of conventional echocardiography performed with the dog in the standing position was at least as good as that obtained with dog in lateral recumbency for most measured variables. Single measurements of each variable may be sufficient for trained observers examining dogs that do not have arrhythmia. The standing position should be used, particularly for stressed or dyspnoeic dogs.

\section{ECHOCARDIOGRAM OF CARDIAC PATIENTS}

Echocardiographic evidence of myocardial failure include decreased fractional shortening, decreased septal and left ventricular free wall percent systolic thickening, 
increased end point septal variation (EPSS), increased left atrial systolic diameter, increased LA/Ao ratio, and decreased aortic excursion. Bonagura et al. (1985) diagnosed congenital heart defects by echocardiography and found it superior to other available non-invasive studies in the recognition and assessment of malformation of the heart. Most frequently encountered cardiac malformations that can be diagnosed include leftto- right shunts, like atrial septal defect, ventricular septal defect, and patent ductus arteriosus; ventricular outflow obstructions like subaortic stenosis and pulmonic valve stenosis; insufficiency of the mitral or tricuspid valves owing to atrioventricular valve dysplasia; complex lesions like teratology of Fallot and reversed patent ductus arteriosus. DeMadron et al. (1985) studied normal and paradoxical ventricular septal motion in the dogs and suggested that abnormalities in ventricular septal motion should cause a clinician to suspect right volume and pressure overload.

Echocardiographic features of pericardial effusion echo-free separation of parietal and visceral pericardium, dampening of parietal pericardial motion, exaggerated or paradoxical motion of intracardiac structures and thickened epicardial echoes (Gugjoo et al., 2013c; Bonagura and Pipers, 1981). Bonagura and Frank (1983) studied valvular lesions in other disease conditions. Studies on valvular endocarditis in a dog, cow and horse revealed following features of valvular lesions - irregular thickening of valves, multiple linear echoes in aortic root, diastolic prolapse of aortic vegetations and diastolic fluttering of aortic valves. In the same study consequences of aortic regurgitation were also elaborated as - left ventricular dilatation, diastolic fluttering of mitral valve, premature closure of mitral valve and left ventricular hyperkinesias.

\section{ECHOCARDIOGRAPHIC STUDY IN DIFFERENT DOG BREEDS}

The echocardiographic values show significant breed variations and it is important to know the normal echocardiographic values for each breed of dog (Jacobson et al., 2013; Gugjoo, 2011). Therefore, stress has given for breed specific studies as echocardiographic reference ranges derived from some breed of dog may be misleading to other breed of dog (Snyder et al., 1995). The variation in echocardiographic values among different breeds may be due to variation in thoracic shape leading to the variation in the direction of the ultrasound beam. Table 1 presents the breeds for which the reference ranges of M-mode echocardiographic measurements have been standardised.

The major differences that are being observed in dog breeds are related to the left ventricular dimensions or the systolic functional parameters. The larger left ventricular dimensions has been reported in athletic breeds viz. Whippets (Bavegems et al., 2007), Greyhounds (Page et al., 1993), Alaskan Sled Dogs (Stepien et al., 1998) and border Collies. This is quite obvious as the heart size is a key determinant of cardiac output, which in turn determines the exercise performance in athletic breeds. To consider it only due to the exercise, it may not be justifiable as even the dogs which do not have daily active training like Border Collies still have the higher values for the left ventricular parameters (Jacobson et al., 2013). It is also unlikely that the larger left ventricular diameters in Border Collies may arise due to slower heart rate, as no correlation between heart rate and chamber size was found. With respect to the fractional shortening (FS), usually smaller values are being observed in athletic dogs like the Border Collies, Whippets, Greyhounds, etc. (Jacobson et al., 2013).

\section{CLINICAL APPLICATIONS}

LVID parameter is of great help in the direct assessment of cardiomyopathies. In case of hypertrophic cardiomyopathy, left ventricular internal diameter decreases in both systole and diastole while in dilation cardiomyopathy, both these parameters increase (Boon, 1998). Unlike ventricles, left atrium increases in size in response to both pressure and volume overload. For confirmatory diagnosis, primary abnormality which is causing the increase in atrial pressure and volume should be identified (Kienle, 1998). Absolute increase in the left atrial diameter is not confirmatory of left atrial dilation and left atrium to aortic root ratio (LAD/AoD) is a better parameter to assess this abnormality. Instead of going for comparison of absolute measurement in a normogram or with a regression equation, this ratio can be solely relied upon to assess left atrial enlargement. A significant increase in left atrial dimension results in a greater left atrial to aortic root ratio with advancing age, as aortic root significantly diminished with age (Vollmar, 1999).

Decreased systolic function evidenced by reduced fractional shortening (FS\%) and ejection fraction ( $<45 \%)$ is the most commonly used clinical measurement of the left ventricular systolic function and is considered a good indicator of ventricular compliance and contractility (Voros et al., 2009). Fractional shortening is an important parameter to distinguish between hypertrophic and dilated cardiomyopathy (Borgarelli et al., 2007). The three conditions that affect the fractional shortening are preload, afterload and contractility and each one of these may act individually or together to affect the FS (Boon, 1998). Ejection fraction (EF) is quite constant in mammals ranging in size from a rat to horses (Riedesel and Knight, 2005).

Clinical significance of EPSS lies in the fact that during the left ventricular or left atrial dilation, an increase in the value of this parameter is seen as the mitral valve is pushed more posterior in these conditions (Feigenbaum, 
Table 1. The breeds for which the reference ranges of M-mode echocardiographic measurements have been standardised.

\begin{tabular}{cll}
\hline S/N & Breed & Reference(s) \\
\hline 1 & English Pointer & Sisson and Schaeffer (1991) \\
\hline 2 & Beagle & Crippa et al. (1992) \\
3 & $\begin{array}{l}\text { Miniature Poodle, Welsh Corgi, Afghan Hound } \\
\text { and Golden Retriever }\end{array}$ & Morrison et al. (1992) \\
\hline 4 & Greyhound & Page et al. (1993), Snyder et al. (1995) \\
\hline 5 & Spanish Mastiff & Bayon et al. (1994) \\
\hline 6 & Boxer & Herrtage (1994) \\
\hline 7 & New Foundland, Wolfhound and Great Dane & Koch et al. (1996) \\
\hline 8 & Whippet, Italian Greyhound and Greyhound & DellaTorre et al. (2000) \\
\hline 9 & Bull Terrier & O'Leary et al. (2003) \\
\hline 10 & Karabash & Kayar and Uysal (2004) \\
\hline 11 & German Shepherd & Kayar et al. (2006), Muzzi et al. (2006) \\
\hline 12 & Whippet & Bavegems et al. (2007) \\
\hline 13 & Indian Spitz & Saxena (2008) \\
\hline 14 & Hungarian breed & Voros et al. (2009) \\
\hline 15 & Indonesian mongrel & Noviana et al. (2011) \\
\hline 16 & Labrador Retriever & Gugjoo (2011) \\
\hline 17 & Border Collies & Jacobson et al. (2013) \\
\hline
\end{tabular}

1981). However, EPSS is only a qualitative indication of the left ventricular function. It should be noted that a normal EPSS value might also occur in the presence of severe cardiac disease. It is a simple measurement, which if altered, should alert the examiner to the possibility of cardiac disease (Kirberger, 1991). Moreover, excessive EPSS correlates well with decreased ejection fraction, although EF may be subnormal while the EPSS remains normal (Massie et al., 1977).

M-mode echocardiography is limited to quantitative measurements of the chamber walls or internal dimensions and cannot be used to measure velocity or the direction of blood flow. However, it can be combined with contrast or colour-coded Doppler studies for accurate timing of flow events (Bonagura and Blissitt, 1995). Its variables are usually subjected to change and needs experienced sonologist to diagnose any condition. It is helpful diagnostic technique when employed with other diagnostic modalities (Gugjoo et al., 2013b).

\section{REFERENCES}

Bavegems V, Duchateau L, Sys SU, Rick AD (2007). Echocardiographic reference values in whippets. Vet. Radiol. Ultrasound 48:230-238.

Bayon A, Fernandezdel Palacio MJ, Montes AM (1994). M-mode echocardiographic study in growing Spanish mastiffs. J. Small Anim. Pract. 35:473-479.

Bishop VS, Horwitz HL, Stone HL (1969). Left ventricular internal diameter and cardiac function in conscious dogs. J. Appl. Physiol.
27:619-623.

Bonagura JD (1983). M-Mode echocardiography- Basic principles. Vet. Clinics of North America: Small anim. Pract. 13:299-319.

Bonagura JD, O'Grady MR, Herring DS (1985). EchocardiographyCongenital Heart Disease. Veterinary Clinics of North America: Small Anim. Pract. 15:1195-1208.

Bonagura JD, Pipers FS (1981). Echocardiographic features of pericardial effusion in dogs. J. Am. Vet. Med. Assoc. 179:49-56.

Bonagura JD, Schober KE (2009). Can ventricular function be assessed by echocardiography in chronic canine mitral valve disease? J. Small Anim. Pract. 50 (Suppl 1):12-24.

Boon JA (1998). Manual of Veterinary Echocardiography. Williams and Wilkins, Baltimore, USA.

Boon JA, Wingfield WE, Miller CW (1983). Echocardiographic indices in normal dog. Vet. Radiol. 24:14-221.

Borgarelli M, Tarducci A, Zanatta R, Haggstrom J (2007). Decreased systolic function and inadequate hypertrophy in large and small breed dogs with chronic mitral valve insufficiency. J. Vet. Intern. Med. $21: 61-67$

Brown DJ, Rush JE, MacGregor J, Ross JN Jr, Brewer B, Rand WM (2003). M-mode echocardiographic ratio indices in normal dogs, cats, and horses: A novel quantitative method. J. Vet. Internal Med. 17:653-662.

Calvert CA, Brown J (1986). Use of M- mode echocardiography in the diagnosis of congestive cardiomyopathy in Doberman pinschers. $\mathrm{J}$. Am. Vet. Medical Assoc. 189:293-297.

Chetboul V, Tidholm A, Nicolle A, Sampedrano CC, Gouni V, Pouchelon JL, Lefebvre HP, Concordet D (2005). Effects of animal position and number of repeated measurements on selected twodimensional and $\mathrm{M}$-mode echocardiographic variables in healthy dogs. J. Am. Vet. Med. Assoc. 227:743-747.

Cornell CG, Kittleson MD, Della Torre PK (2004). Allometric scaling of $\mathrm{M}$ - mode cardiac measurements in normal adult dogs. J. Vet. Internal Med. 18:311-21.

Crippa L, Ferro E, Melloni E, Brambilla, Cavalletti E (1992). Echocardiographic parameters and indices in normal Beagle dogs. Lab. Anim. 26:190-195.

Curie P, Curie J (1880). Development, par pression de l'electricite 
polarize dans les cristaux hemiedres a faces inclines. Comptes Rendus 92:291-295.

DellaTorre PK, Kirby AC, Church DB, Malik R (2000). Echocardiographic measurements in Greyhound, Whippet and Italian Greyhound dogs with similar conformation but different sizes. Aus. Vet. J. 78:49-55.

DeMadron E, Bonagura JD, O'Grady MR (1985). Normal and paradoxical ventricular septal motion in the dog. Am. J. Vet. Res. 46:1832-1841.

Dennis MO, Nealeigh RC, Pyle RL (1978). Echocardiographic assessment of normal and abnormal valvular function in Beagle dogs. Am. J. Vet. Res. 39:1591-1598.

Edler I, Hertz CH (1954). Use of ultrasonic reflectoscope for continuous recording of movements of heart walls. Kung. Fysiogr. Sallsk. Lund. Forth 24:40-46.

Feigenbaum H (1981). Echocardiography. 3rd ed. Lea and Febiger, Philadelphia. USA.

Franklin TD, Weyman AE, Egenes KM (1977). A closed chest model for cross-sectional echocardiographic study. Am. J. Physiol. 233:H417H419.

Frazin L, Talano JW, Stephanides L, Loeb HS, Kopel L, Gunnar RM (1976). Esophageal echocardiography. Circulation 54: 102-108.

Goncalves AC, Orton EC, Boon JA, Salman MD (2002). Linear, logarithmic and polynomial models of $\mathrm{M}$-mode echocardiographic measurements in dogs. Am. J. Vet. Res. 63:994-99.

Gugjoo MB (2011). Determination of radiographic, electrocardiographic, echocardiographic and haemato-biochemical cardiac reference values in Labrador retriever (M.V.Sc. Thesis) I.V.R.I-Izatnagar, India.

Gugjoo MB, Hoque M, Saxena AC, Zama MMS (2013a). Radiographic, electrocardiographic and echocardiographic features of dilatation cardiomyopathy in dogs. Indian Vet. J., 90 (12): 54-56.

Gugjoo MB, Hoque M, Saxena AC, Zama MMS and Yatoo MI (2013b). Pericardial effusion in dogs: diagnostic features of radiography, electrocardiography, and echocardiography. Int. J. Cur. Res. (InPress).

Gugjoo MB, Saxena AC, Hoque M, Mahendran K and Zama MMS (2013c). Pericardial effusion in a Labrador retriever dog. Asian J. Anim. Sci. (InPress).

Herrtage M (1994). Echocardiographic measurements in the normal Boxer. In: Proc Eur Soc Vet Intern Med. $4^{\text {th }}$ Ann Cong., P. 172.

Jacobs G, Knight DH (1985). M-mode echocardiographic measurement in non-anesthetized healthy dogs and cats: Effects of body weight, heart rate and other variables. Am. J. Vet. Res. 46:1705-1711.

Jacobs G, Mahjoob K (1988a). Influences of alterations in heart rate on echocardiographic measurements in dogs. Am. J. Vet. Res. 49:548552.

Jacobs G, Mahjoob K (1988b). Multiple regression analysis using body size and cardiac cycle length in predicting echocardiographic variables in dogs. Am. J. Vet. Res. 49:1290-1294.

Jacobson JH, Boon JA, Bright JM (2013). An echocardiographic study of healthy Border Collies with normal reference ranges for the breed. J. Vet. Cardiol. 15: 123-130.

Kayar A, Gonul R, Or ME, Uysal A (2006). M-mode echocardiographic parameter and indices in the normal German shepherd Dog. Vet. Rad. Ultrasound 47:482-486.

Kayar A, Uysal A (2004). Determination of cardiac reference parameters using M-mode and two-dimensional echocardiographic techniques in adult Karabash dog. Turk. J. Vet. Anim. Sci. 28:39-46.

Kiedel WD (1950). Uber eine method zur registrierung der Volumanderungen des Herzens am Menschen. Z Kreislaufforsch 39:257.

Kienle RD (1998). In: Kittleson, MD and Kienle, RD (eds.). Small Animal Cardiovascular Medicine. Mosby Inc., St. Louis, pp. 402-412.

Kittleson MD (1998). In: Kittleson MD and Kienle RD (eds.). Small Animal Cardiovascular Medicine. Mosby Inc., St. Louis, pp. 218-230.

Kirberger, RM (1991). Mitral valve E point to ventricular septal separation in the dog. J S. Afr. Vet. Assoc., 62:163-166.

Koch J, Pederson HD, Jensen AL (1996). M-mode echocardiographic diagnosis of dilated cardiomyopathy in giant breed of dogs. J. Am. Vet. Med. Assoc. 43:297-304.

Lombard CW (1984). Normal values of canine $M$ mode echocardiogram. Am. J. Vet. Res. 45: 2015-2018.
Mashiro I, Nelson RR, Cohn JN, Franciosa JA (1976). Ventricular dimensions measured non-invasively by echocardiography in the awake dog. J. Appl. Physiol. 41:953- 959.

Massie BM, Schiller NB, Ratshin RA, Parmley WM (1977). Mitral-septal separation: new echocardiographic index of left ventricular function. Am. J. Cardiol. 44:1332-1338.

Morrison SA, Moise NS, Scarlett J (1992). Effect of breed and body weight on echocardiographic values in four breeds of dogs of different somatotypes. J. Vet. Intern. Med. 6:220-224.

Muzzi RAL, Muzzi LAP, Arauzo RB, Cherem M (2006). Echocardiographic indices in normal German shepherd dog. J. Vet. Sci. 7:193-198.

Noviana D, Paramitha D, Wulansari R (2011). Motion mode and two dimensional echocardiographic measurements of cardiac dimensions of Indonesian mongrel dogs. Hayati J. Biosci. 18:1-5.

O' Grady MR, Bonagura JD, Powers JD, Herrings DS (1986). Quantitative cross-sectional echocardiography in the normal dog. Vet. Radiol. 27:34-49.

O'Leary CA, Mackay BM, Taplin RH, Atwell RB (2003). Echocardiographic parameters in 14 healthy English Bull terriers. Aus. Vet. J. 81:535-542.

Oyama AM (2004). Advances in echocardiography. Veterinary Clinics of North America: Small Anim. Pract. 34:1083-1104.

Oyama AM, Sisson DD (2005). Assessment of cardiac chamber size using anatomical $M$ - mode. Vet. Radiol. Ultrasound 46:331-336.

Page A, Edmunds G, Atwell RB (1993). Echocardiographic values in Greyhounds. Aus. Vet. J. 70:361-364.

Pipers FS, Bonagura JD, Hamlin RL, Kittleson M (1981). Echocardiographic abnormalities of mitral valve associated with left sided heart diseases in dogs. J. Am. Vet. Med. Assoc. 179:580-586.

Riedesel, DH, Knight, DH (2005). In: Reece, WO (eds). Duke's Physiology of Domestic Animals. Panima Publishing Corporation, New Delhi, 230.

Rishniw M, Erb HN (2000). Evaluation of four 2-dimensional echocardiographic methods of assessing left atrial size in dogs. J. Vet. Internal Med. 14:429-35.

Saxena AC (2008). Studies on the measurement of reference ranges of echocardiographic indices and related cardiac parameters in Indian Spitz breed of the dogs (M.V.Sc. Thesis) I.V.R.I-Izatnagar, India.

Schober KE, Baade H (2000). Comparability of left ventricular m-mode echocardiography in dogs performed in long-axis and short-axis. Vet. Radiol. Ultrasound 41:543-549.

Sisson D, Schaeffer D (1991). Changes in linear dimensions of the heart relative to body weight as measured by M-mode echocardiography in growing dogs. Am. J. Vet. Res. 52:1519-1596.

Snyder PS, Sato T, Atkins CE (1995). A comparison of echocardiographic indices of the non-racing healthy greyhound to reference values from other breeds. Vet. Radiol. Ultrasound 36:387392.

Stepien RL, Hinchcliff KW, Constable PD, Olson J (1998). Effect of endurance training on cardiac morphology in Alaskan sled dogs. J. Appl. Physiol. 85:1368-1375.

Teicholz LE, Kreulen T, Herman MV, Gorlin R (1976). Problems in echocardiographic volume determinations: echocardiographicangiographic correlations in presence or absence of asynergy. Am. J. Cardiol., 37:7-11.

Thomas WP (1984). Two-dimensional real time echocardiography in the dog: Techniques and anatomic validation. Vet. Radiol. 25:50-64.

Thomas WP, Gaber CE, Jacobs GJ (1993). Recommendations for standards in transthoracic two-dimensional echocardiography in the dog and cat. J. Vet. Int. Med. 7:247-252.

Vollmar AC (1999). Echocardiographic measurements in Irish wolfhound: reference values for the breed. J. Am. Anim. Hosp. Assoc. 35:271-277.

Voros K, Hetyey C, Reiczegel J, Czirok GN (2009). M-mode and two dimensional echocardiographic reference values for three Hungarian dog breeds. Acta Veterinaria Hungarica 57:217-227.

Weyman AE (1982). Cross-sectional echocardiography. Lea and Febiger, Philadelphia.

Wild JJ, Crawford HD, Reid JM (1957). Visualization of excised human

heart by means of reflected ultrasound or echocardiography. Am. Heart J. 54:903-906. 
Wyatt HL, Haendchen RV, Meearbaum S (1980). Assessment of quantitative methods for two-dimensional echocardiography. Am. J. Cardiol. 52:396-401.

Yamamura H, Etoh T, Satoh T, Yamazaki R, Onoda S, Terada T (1977). Application of echocardiography in dogs-analysis of echocardiograms of ventricular walls and values. Adv. Anim. Electrocard. 10:33-44. 